\title{
Biomasa acústica y distribución del jurel Trachurus murphyi en el Perú
}

\author{
Acoustic biomass and distribution of Jack mackerel Trachurus murphyi in Peru
}

\author{
Marceliano Segura y Aníbal Aliaga
}

Instituto del Mar del Perú, esquina Gamarra y General Valle S/N Chucuito, Callao, Perú.

Email Marceliano Segura: msegura@imarpe.gob.pe
Citación:

Segura M. \& A. Aliaga. 2013. Biomasa acústica y distribución del jurel Trachurus murphyi en el Perú. En: Csirke J., R. Guevara J., R. Guevara-Carrasco \& M. Espino (Eds.). Ecología, pesquería y conservación del jurel (Trachurus murphyi) en el Perú. Rev. peru. biol. número especial 20(1): 087-096 (Septiembre 2013)

\section{Resumen}

Se analizan los resultados de las evaluaciones hidroacústicas del recurso jurel Trachurus murphyi Nichols 1920 realizadas en aguas peruanas entre 1983-2012. Desde 1983 se incluyó al T. murphyi como especie de estudio durante los cruceros de evaluación de recursos pelágicos ejecutados por el Instituto del Mar del Perú. Debido al énfasis en la estimación de biomasa de la anchoveta Engraulis ringens y de la sardina Sardinops sagax cuando esta última es más abundante, los cruceros se llevan a cabo durante el verano austral y las áreas de evaluación están circunscritas a las zonas más costeras hasta $100 \mathrm{mn}$, con sólo algunas exploraciones en otras estaciones y hasta 200 millas. El máximo valor de biomasa de 8.51 millones de toneladas de T. murphyi en aguas peruanas estimado con las evaluaciones hidroacústicas fue encontrado durante el crucero realizado en otoño (marzo-mayo) de 1983. En los años siguientes los estimados de biomasa acústica fluctuaron entre 180 mil toneladas en 1985 y otro máximo de 8.47 millones de toneladas en 1993, para luego disminuir gradualmente hasta un mínimo de 1239 t en 2010, con una ligera recuperación en los años 2011 y 2012 . El área de distribución de T. murphyi fue muy fluctuante en todo el periodo observado.

Palabras clave: Jurel, biomasa acústica, recursos pelágicos

\section{Abstract}

This paper analyzes the results of acoustic stock assessment surveys for Jack mackerel Trachurus murphy Nichols 1920 conducted in Peruvian waters between 1983 and 2012. Since 1983 Jack mackerel T. murphyi was included as a target species to be assessed during the pelagic stock assessment surveys executed by the Peruvian Institute of Marine Research. Due to the emphasis on the assessment of anchovy Engraulis ringens and sardine Sardinops sagax when this species was abundant, these surveys usually took place during the austral summer and only covered the first 100 nautical miles from the coast, and only occasional surveys were conducted in other seasons or surveyed as far as $200 \mathrm{~nm}$ offshore. A maximum biomass of 8.51 million t of T. murphyi in Peruvian waters estimated through acoustics during a survey carried out in autumn (March-May) 1983. In the following years acoustic biomass estimates ranged between 180 thousand $t$ in 1985 and another maximum of 8.47 million $t$ in 1993 , to then decreased gradually to a minimum of $1239 \mathrm{t}$ in 2010 , with a slight recovery in 2011 and 2012. The areas of distribution of T. murphyi have been fluctuating markedly throughout the observed period.

Keywords: Jack mackerel, acoustic biomass, pelagic resources.

\section{Introducción}

Trachurus murphy Nichols 1920 es una especie pelágica transzonal que presenta una amplia distribución asociada a las fluctuaciones de distinta escala en el ambiente del Pacífico Sudoriental, las que han dado lugar también a cambios en el largo plazo en la abundancia y distribución de los recursos pesqueros pelágicos y en sus pesquerías (Jordán 1983, Zuta et al. 1983, Serra 1983 y 1991, Csirke 1995). En el Perú, en algunas áreas y bajo ciertas condiciones oceanográficas T. murphyi se encuentra compartiendo las mismas áreas con otras especies como la caballa Scomber japonicus, anchoveta $E$. ringens y la sardina $S$. sagax.

En este trabajo se presentan los resultados de las estimaciones de biomasa y áreas de distribución de T. murphyi con el método hidroacústico obtenidos de los cruceros de
Publicado online: $\quad 11 / 10 / 2013$ Publicado impreso: $15 / 10 / 2013$ 
evaluación de los principales recursos pelágicos realizados entre 1983 - 2012 por el Instituto del Mar del Perú. La evaluación directa del T. murphyi por hidroacúastica se efectúa en el Perú como parte de la evaluación del principal recurso pesquero nacional que es la anchoveta E. ringens. Por ello estos cruceros de evaluación se realizan por lo general en verano y sólo abarcaron la franja costera hasta 100 millas náuticas $(\mathrm{mn})$ de distancia de la costa. Ocasionalmente se han ejecutado este tipo de cruceros en otras estaciones del año y en algunos casos se llegó hasta las $200 \mathrm{mn}$ de la costa, como los cruceros hechos en la primavera de 1986 y verano de 1990 (Ganoza 1998). Por ello se considera que los estimados de las biomasas de T. murphyi por el método hidroacústico representan sólo una fracción de la biomasa total disponible en toda el área potencial de su distribución frente a la costa peruana.

\section{Material y métodos}

Durante el periodo 1983 - 2012, los cruceros para evaluar la biomasa acústica y la distribución de recursos pelágicos ejecutados por el Instituto del Mar del Perú han mantenido en general la misma cobertura espacial desde el extremo norte al extremo sur del dominio marítimo peruano, siguiendo transectos sistemáticos paralelos (Johannesson \& Robles 1977, MacLennan \& Simmonds 1992) perpendiculares a la costa, con una separación de 12 a $17 \mathrm{mn}$ entre transectos. Estos cruceros se realizaron a bordo de los buques de investigación científica (BIC) SNP-1, Humboldt, Olaya y SNP-2. Además, en 1990 se realizó una evaluación conjunta con el R/V Nansen (Ex-URSS).

La identificación de cada crucero se hace mediante un código compuesto de dos letras seguidas de cuatro o seis números. Las dos primeras letras "Cr" indican que se trata de un crucero, los dos números siguientes indican el año en que se realizó el crucero y los dos o cuatro números siguientes indican el mes o meses en que se realizó el mismo. Es así que, por ejemplo "Cr.1102-04" identifica el crucero realizado el año 2011 entre los meses de febrero y abril.

A lo largo del periodo de estudio se utilizaron equipos científicos vigentes en su época, los que siempre se mantuvieron actualizados. En los primeros años se emplearon equipos analógicos tales como las ecosondas científicas SIMRAD de modelos EK y ecointegradores Hewlett Packard, y en los últimos años se vienen empleando equipos digitales SIMRAD modelo EK60 en frecuencia de trabajo 38, 120 y $200 \mathrm{kHz}$, y con software de procesamiento y ecointegración Echoview.

Al inicio de cada exploración se efectúa la calibración del sistema de evaluación hidroacústico para determinar la constante del instrumento $\left(\mathrm{C}_{\mathrm{i}}\right)$. A partir de 1992 se empezó a calibrar con blanco estándar (Foote 1987, SIMRAD 1992) utilizando esferas de cobre de $23 \mathrm{~mm}(38 \mathrm{kHz}), 60 \mathrm{~mm}(120 \mathrm{kHz})$ y $13.7 \mathrm{~mm}(200$ $\mathrm{kHz}$ ). Asimismo, cuando la evaluación se realiza con más de una embarcación y con el objeto de estandarizar el rendimiento del sistema acústico, se efectúa una intercalibración entre las embarcaciones participantes, navegando en forma paralela muy cerca una de la otra y ejecutando ecointegración simultánea.

Teniendo en cuenta la fuerza del eco del blanco (TS) específica para cada especie, el procedimiento de cuantificación durante el muestreo acústico se hace a través de un proceso de ecointegración por Unidades Básicas de Muestreo (UBM). Se integra la señal de los ecos por rangos de distancia de $1 \mathrm{mn}$, a velocidad aproximada de 10 nudos. La ecointegración consiste en una integración del area de retrodispersión transversal de los ecos recibidos, también conocida como back-scattering strength area. Los resultados de la ecointegración se dan clásicamente en $\mathrm{m}^{2}$ por $\mathrm{mn}$ (Simmonds \& MacLennan 2005),

Durante las exploraciones acústicas se realizan operaciones de pesca para la comprobación de los ecotrazos y para realizar muestreos biológicos. Las operaciones de pesca se realizan con red de arrastre pelágica $124 / 1800 \mathrm{~mm}$ de poliamida (PA), con una longitud total de $73 \mathrm{~m}$ y una abertura vertical promedio de $12 \mathrm{~m}$. Esta red tiene un copo interno de tamaño de malla de $12 \mathrm{~mm}$ y los lances tienen una duración efectiva entre $15 \mathrm{y}$ 30 minutos, dependiendo de la densidad del número de cardúmenes. El comportamiento de la red de pesca es monitoreado con una netsonda.

La separación de los valores integrados por especies se hace por reconocimiento directo del cardumen y/o asignación de valores, para lo cual se toman en cuenta los resultados de las operaciones de pesca (Simmonds \& MacLennan 2005). Luego de hacer la separación de los valores integrados $\left(\mathrm{m}^{2} / \mathrm{mn}\right)$ por especie, se determinan las áreas de distribución y concentración con el software SURFER mediante el método de interpolación kriging y contorneo (Gutiérrez 1997). Se han establecido cuatro categorías de concentración: muy disperso $\left(0-10 \mathrm{~m}^{2} / \mathrm{mn}\right)$, disperso $\left(>10-100 \mathrm{~m}^{2} / \mathrm{mn}\right)$, denso $\left(>100-1000 \mathrm{~m}^{2} / \mathrm{mn}\right)$ y muy denso $\left(>1000 \mathrm{~m}^{2} / \mathrm{mn}\right)$.

La biomasa es calculada por áreas isoparalitorales que tienen una dimensión de $0.5^{\circ}$ de latitud por $10 \mathrm{mn}$ de ancho siguiendo un trazado paralelo a la línea de costa. Este es un sistema propio desarrollado por el IMARPE en los ańos 70 y luego fue definido en formato digital (Gutiérrez \& Peraltilla 1999). Previamente los datos son tratados con el software MapInfo y los cálculos se hacen con el software R (http://www.r-project.org/).

A partir de 1992 se siguió un sistema de procesamiento para los estimados de la biomasa algo diferente al seguido en ańos anteriores. Entre 1983 y 1991 la biomasa se obtenía del producto del área del estrato, los valores integrados de la especie en el estrato y una constante de calibración estimada a partir de mediciones electrónicas, acústicas y con peces vivos en jaula (Johannesson \& Vílchez 1981). De 1992 a la fecha se sigue el mismo procedimiento sólo que la constante de calibración se estima a partir de la calibración con blanco estándar y la relación fuerza de blanco (TS)/longitud (L) del jurel (TS/L).

Entre 1992 y 1997, se trabajó con ecosonda de $38 \mathrm{kHz}$ y para calcular la fuerza de blanco (TS) se utilizó la ecuación (Foote 1987):

$$
\mathrm{TS}=20 \log \mathrm{L}-71.9 \mathrm{~dB}
$$

A partir de 1998 se trabajó con ecosonda de $120 \mathrm{kHz}$ y se uso la siguiente ecuación para calcular la fuerza de blanco (Gutiérrez et al. 1999, Castillo et al. 2009):

$$
\mathrm{TS}=20 \log \mathrm{L}-70.8 \mathrm{~dB}
$$

De este modo, la biomasa por área isoparalitoral, que esta referida a distancia de la costa, se calcula de acuerdo a la siguiente fórmula, donde el área de retrodispersión transversal $(\sigma)$, la densidad de peces $(\rho)$, el número de individuos $(n)$ y la biomasa en el estrato es calculada de: 


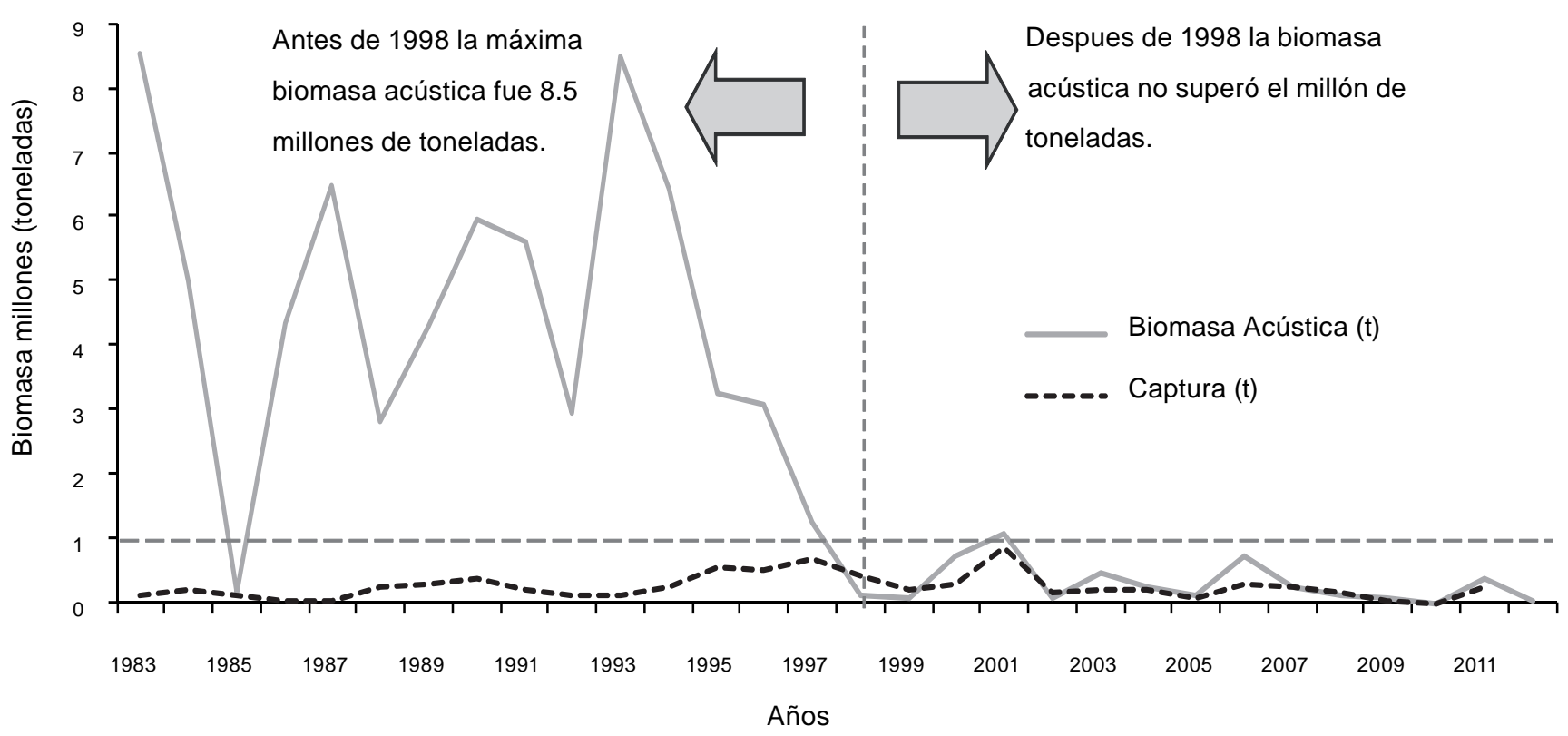

Figura 1. Biomasa estimada por hidroacústica y desembarques de jurel T. murphyi en toneladas, años 1983-2012.

Figure 1. Jack mackerel T. murphyi acoustic biomass estimates and landings in tons, years 1983-2012.

$$
\begin{aligned}
& \sigma=4(\pi) 10^{\mathrm{TS} / 10} \\
& \rho=10000 \mathrm{C}_{\mathrm{i}} \mathrm{S}_{\mathrm{a}} / \sigma \\
& \mathrm{n}=\rho \mathrm{A}
\end{aligned}
$$

Biomasa $=n^{*} \mathrm{~W} / 1000000$

donde,

$$
\begin{array}{ll}
\mathrm{C}_{\mathrm{i}} & : \text { Constante del instrumento } \\
\rho & : \text { Densidad peces } / \mathrm{m}^{2} \\
\mathrm{~A} & : \text { Área isoparalitoral }\left(\mathrm{mn}^{2}\right) \\
\mathrm{S}_{\mathrm{a}} & : \text { valor ecointegrado promedio }\left(\mathrm{m}^{2} / \mathrm{mn}^{2}\right) \\
\mathrm{W} & : \text { peso promedio del jurel }(\mathrm{g}) \\
n & : \text { número de peces }
\end{array}
$$

Los límites de confianza fueron determinados según lo propuesto por Bazigos (1975):

$$
L C=B \pm 1.96 * \sqrt{\sum\left[\left(\frac{s_{j}^{2}}{n_{j}}\right) *\left(A_{j}^{2}\right)\right]} /\left[\sum A_{j}\right]^{2} /\left[\sum\left[N A S C_{j} * A_{j}\right] / \sum\left(A_{j}\right)\right] * 100
$$

Donde:

B: biomasa o abundancia total de la evaluación (toneladas)

$S_{j}^{2}$ : varianza del estrato $j$

$n_{j}$ : número de UBMs en el j-ésimo estrato.

$A$ : área del $\mathrm{j}$-ésimo estrato $\left(\mathrm{mn}^{2}\right)$

NASC: Coeficiente Náutico de Dispersión Acústica promedio en el j-ésimo estrato

\section{Resultados y discusión}

Biomasa.- Durante el periodo 1983 - 2012 se registraron dos valores máximos en los estimados de biomasa hidroacústica: en el otońo de 1983 (Cr.8303-05) con 8512674 t; y en el verano de 1993 (Cr.9301-03) con 8471096 t (Figura 1, Tabla 1). Después de El Niño 1982-83, en el invierno de 1984 (Cr.8406-09), la biomasa estimada disminuyó a 5001913 t. El proceso de enfriamiento de 1985 originó el desplazamiento longitudinal del frente oceánico, lo cual produjo el alejamiento de T. murphyi a distancias mayores de la costa, fuera del área prospectada (Ganoza 1998), lo que explicaría los bajos estimados de biomasa ese año. En los años siguientes se notó una recuperación en los estimados, hasta alcanzar otro máximo valor en 1993. Los eventos cálidos de 1987-88 y 1992 afectaron la distribución y biomasa de este recurso, dando lugar a desplazamientos hacia el sur. Luego, a partir de 1994, se produjo una disminución paulatina de la biomasa estimada, con ligeras recuperaciones en el 2000-2001, 2006 y 2011.

Con relación a las densidades promedio en función a las áreas de distribución, los mayores valores de áreas de distribución de T. murphyi en la franja costera evaluada se dieron en la década de los 80 y principios de los 90, mientras que los valores más altos de densidad se dieron en los primeros años de la década de los 90 (Tabla 1 y Fig. 2). En general se observo una tendencia a encontrar densidades medias más altas cuando las áreas de $T$. murphyi eran menores y más pegadas a la costa.

Luego de El Nińo de 1992 se observo un incremento de la densidad, que alcanzo sus máximos valores en 1993 y 1994 con 336 y $368 \mathrm{t} / \mathrm{mn}^{2}$ respectivamente, para disminuir a $185 \mathrm{t} / \mathrm{mn}^{2}$ en 1996 en áreas de evaluación semejantes. En años más recientes las densidades y las biomasas acústicas se han mantenido bajas y los valores más bajos de los últimos años se encontraron en 2010, con un ligero repunte en 2011 donde la biomasa estimada por acústica fue de 363 mil toneladas con densidades medio-altas pero reducidas para descender nuevamente en 2012 (Tabla 1).

Las áreas exploradas durante los cruceros científicos de evaluación acústica han cubierto todos los años la franja más costera del mar peruano y es relevante destacar que la extensión de estas áreas se mantuvo más o menos constante durante el período estudiado (Fig. 3). En la Figura 3, también se puede observar que hasta principios de los años 90 (Crucero 9301-03) se encontró T. murphyi en buena parte (casi el 50\%) de las áreas exploradas. En los años siguientes la porción ocupada por las áreas de distribución del T. murphyi disminuyen significativamente, y disminuyen con mayor notoriedad a partir de 2001. 


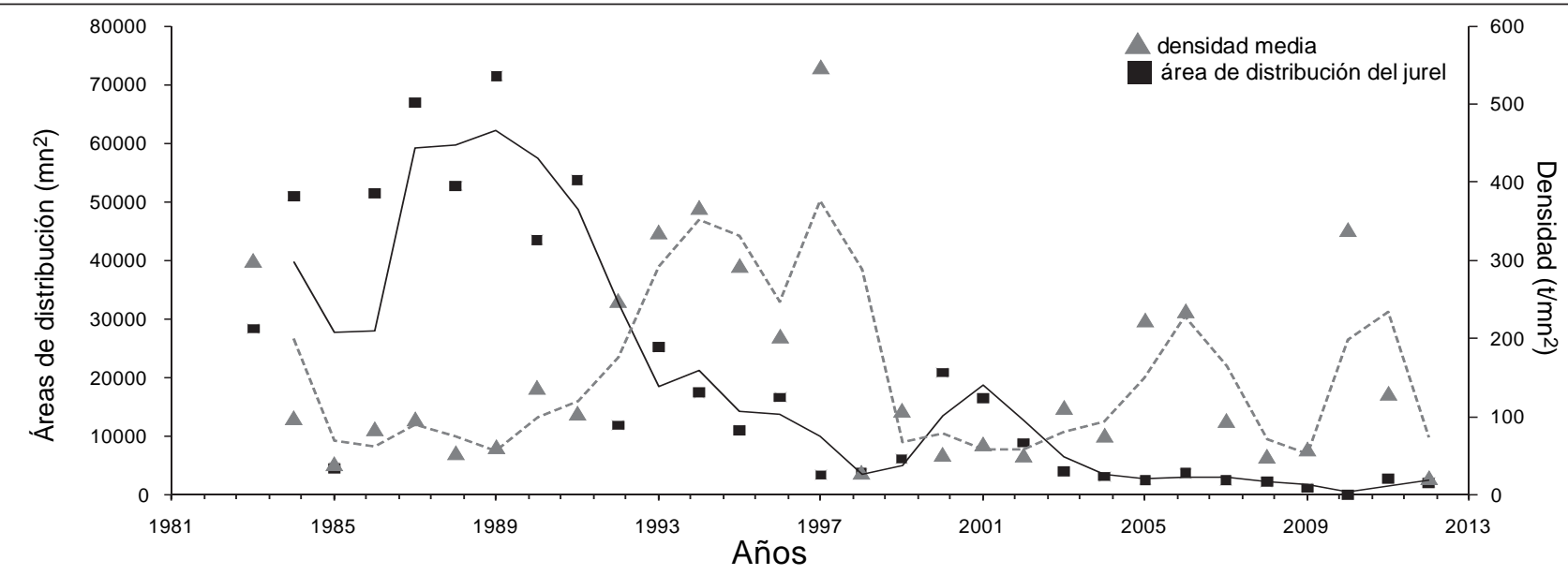

Figura 2. Áreas de distribución $\left(\mathrm{mn}^{2}\right)$ y densidades medias $\left(\mathrm{t} / \mathrm{mn}^{2}\right)$ de jurel $T$. murphyi y sus medias móviles (lineas continua y punteadas) por años, $1983-2012$.

Figure 2. Distribution areas $\left(\mathrm{mn}^{2}\right)$ and mean densities $\left(\mathrm{t} / \mathrm{mn}^{2}\right)$ of Jack mackerel T. murphyi with their moving averages (solid and broken lines), by year, $1983-2012$.

Tabla 1. Información general de los cruceros de evaluación acústica ejecutados entre 1983 y 2012, incluyendo información sobre el área evaluada y las áreas de distribución, biomasa acústica y densidad promedio de jurel T. murphyi estimadas en cada crucero.

Table 1. General information of the acoustic surveys conducted between 1983 and 2012, including information on the surveyed area and the areas of distribution, acoustic biomass and mean densities of Jack mackerel T. murphyi estimated on each survey.

\begin{tabular}{|c|c|c|c|c|c|c|c|}
\hline Año & Crucero & $\begin{array}{c}\text { Área distribución } \\
\text { del jurel }\left(\mathrm{mn}^{2}\right)\end{array}$ & $\begin{array}{c}\text { Área total } \\
\text { evaluada }\left(\mathbf{m n}^{2}\right)\end{array}$ & Biomasa acustica & Densidad & Área jurel & Densidad media \\
\hline 1983 & 8309-12 & 28368 & 66711 & 8512674 & 300.080161 & 10.253017 & 5.70404964 \\
\hline 1984 & 8406-09 & 50922 & 126627 & 5001913 & 98.226955 & 10.8380503 & 4.58728067 \\
\hline 1985 & 8503-05 & 4592 & 76750 & 180259 & 39.2550087 & 8.43207094 & 3.67007905 \\
\hline 1986 & 8604-05 & 51480 & 85973 & 4329874 & 84.1078866 & 10.8489487 & 4.43210034 \\
\hline 1987 & 8708-09 & 66898 & 100227 & 6471955 & 96.7436246 & 11.1109244 & 4.57206443 \\
\hline 1988 & 8805-07 & 52701 & 115813 & 2811590 & 53.3498416 & 10.8723897 & 3.97687101 \\
\hline 1989 & 8907-09 & 71470 & 120712 & 4365164 & 61.0768714 & 11.1770331 & 4.11213326 \\
\hline 1990 & $9002-04$ & 43522 & 94323 & 5971710 & 137.211295 & 10.6810218 & 4.92152204 \\
\hline 1991 & 9106-08 & 53748 & 104269 & 5597492 & 104.143261 & 10.8920617 & 4.64576746 \\
\hline 1992 & 9201-03 & 11893 & 74952 & 2957578 & 248.68225 & 9.38370527 & 5.51617598 \\
\hline 1993 & 9301-03 & 25173 & 100496 & 8471096 & 336.515155 & 10.1335273 & 5.81864319 \\
\hline 1994 & 9401-03 & 17430 & 107606 & 6413607 & 367.963683 & 9.76594814 & 5.90798425 \\
\hline 1995 & $9502-04$ & 11077 & 69444 & 3251207 & 293.509705 & 9.31262617 & 5.6819107 \\
\hline 1996 & $9602-04$ & 16633 & 103683 & 3375866 & 202.961943 & 9.71914395 & 5.31301849 \\
\hline 1997 & 9709-10 & 3452 & 50927 & 1892286 & 548.170915 & 8.14670905 & 6.30658713 \\
\hline 1998 & 9803-05 & 3789 & 50948 & 107236 & 28.3019266 & 8.23985741 & 3.34292988 \\
\hline 1999 & $9902-03$ & 6136 & 89618 & 661714 & 107.846557 & 8.72187945 & 4.68070945 \\
\hline 2000 & 0001-02 & 20841 & 110375 & 1070788 & 51.3779012 & 9.94469711 & 3.93920814 \\
\hline 2001 & 0103-04 & 16492 & 114736 & 1066544 & 64.6699094 & 9.71063821 & 4.16929601 \\
\hline 2002 & 0202-03 & 8869 & 82029 & 446782 & 50.3773522 & 9.0902836 & 3.91954171 \\
\hline 2003 & 0302-03 & 4067 & 98898 & 453597 & 111.543714 & 8.31054888 & 4.71441657 \\
\hline 2004 & 0402-03 & 3155 & 104461 & 239356 & 75.8700393 & 8.05668472 & 4.32902187 \\
\hline 2005 & 0502-04 & 2511 & 104387 & 561535 & 223.607457 & 7.82853715 & 5.40989209 \\
\hline 2006 & 0602-04 & 3736 & 88189 & 879266 & 235.350091 & 8.22576881 & 5.46107416 \\
\hline 2007 & 0702-04 & 2494 & 108580 & 236235 & 94.7294407 & 7.8215585 & 4.55102484 \\
\hline 2008 & 0802-04 & 2245 & 140171 & 109643 & 48.8463125 & 7.71630411 & 3.88867889 \\
\hline 2009 & 0902-03 & 1204 & 89618 & 70074 & 58.1791896 & 7.09377692 & 4.06352772 \\
\hline 2010 & $1002-04$ & 69 & 72690 & 23429 & 339.544435 & 4.2341065 & 5.82760482 \\
\hline 2011 & 1102-04 & 2798 & 89114 & 362777 & 129.6558 & 7.93666016 & 4.86488325 \\
\hline 2012 & $1202-04$ & 2101 & 97636 & 44934 & 21.3848845 & 7.65026568 & 3.06268434 \\
\hline
\end{tabular}




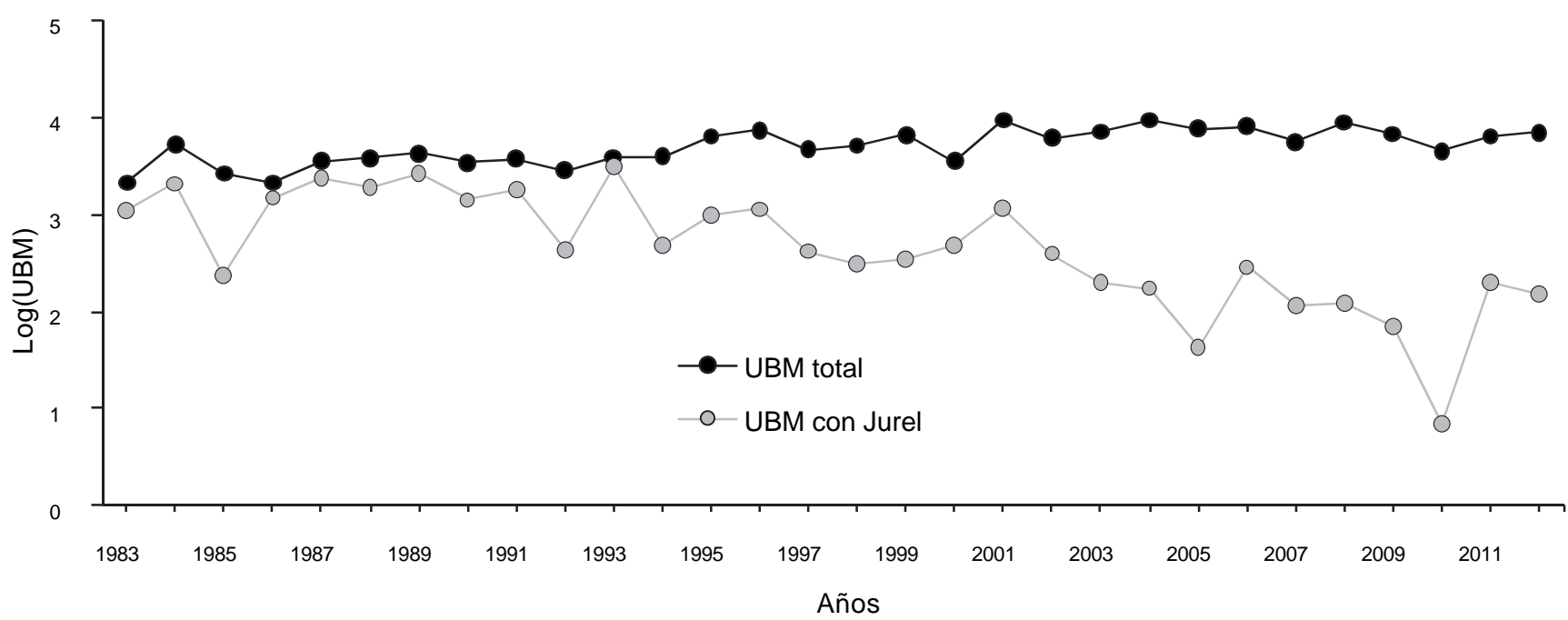

Figura 3. Áreas exploradas durante los cruceros de evaluación de recursos pelágicos (línea negra) y áreas de distribución de jurel T. murphyi (línea gris) entre 1983 y 2012.

Figure 3. Explored areas during the pelagic stock assessment acoustic surveys (black line) and areas of distribution of Jack mackerel $T$. murphyi (gray line). $1983-2012$.

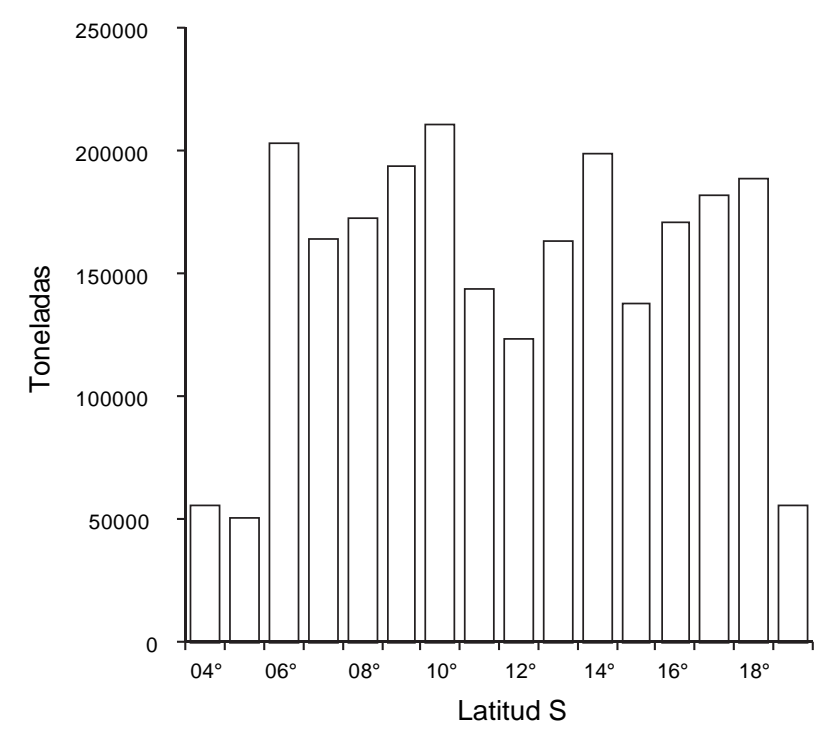

Figura 4. Biomasas promedio de jurel T. murphyi por grados de latitud de la serie $1983-2012$.

Figure 4. Jack mackerel T. murphyi average biomass per latitude degrees for the period $1983-2012$.
Según Barbieri et al. (2004, 2005) el T. murphyi tendría una cierta capacidad de adaptación a las características cambiantes del ecosistema, y en particular a los cambios relacionados con eventos como El Niño y La Niña, y esto podría explicar en parte los cambios en las áreas de distribución y densidad observados. Desde 2000 los cardúmenes de T. murphyi que fueron detectados durante las exploraciones acústicas se encontraban en forma dispersa e individual (no agregados), razón por la cual las densidades fueron por lo general muy bajas.

Distribución.- Entre 1983 y 2012 (Fig. 4), la distribución promedio de la biomasa de T. murphyi por grados de latitud en el mar peruano entre 1983 y 2012 confirma la presencia de esta especie a lo largo de todo el litoral peruano y, especialmente, de los $5^{\circ} \mathrm{S}$ hacia el sur. Esta observación general de la distribución de T. murphyi detectado acústicamente guarda buena relación con la presencia de huevos y larvas de la misma especie en toda la costa del Perú (Ayón \& Correa 2013).

La imagen sinóptica de la variación latitudinal de la biomasa acústica con el tiempo (Fig. 5) muestra como se han dispuesto los principales focos de concentración a lo largo de la costa

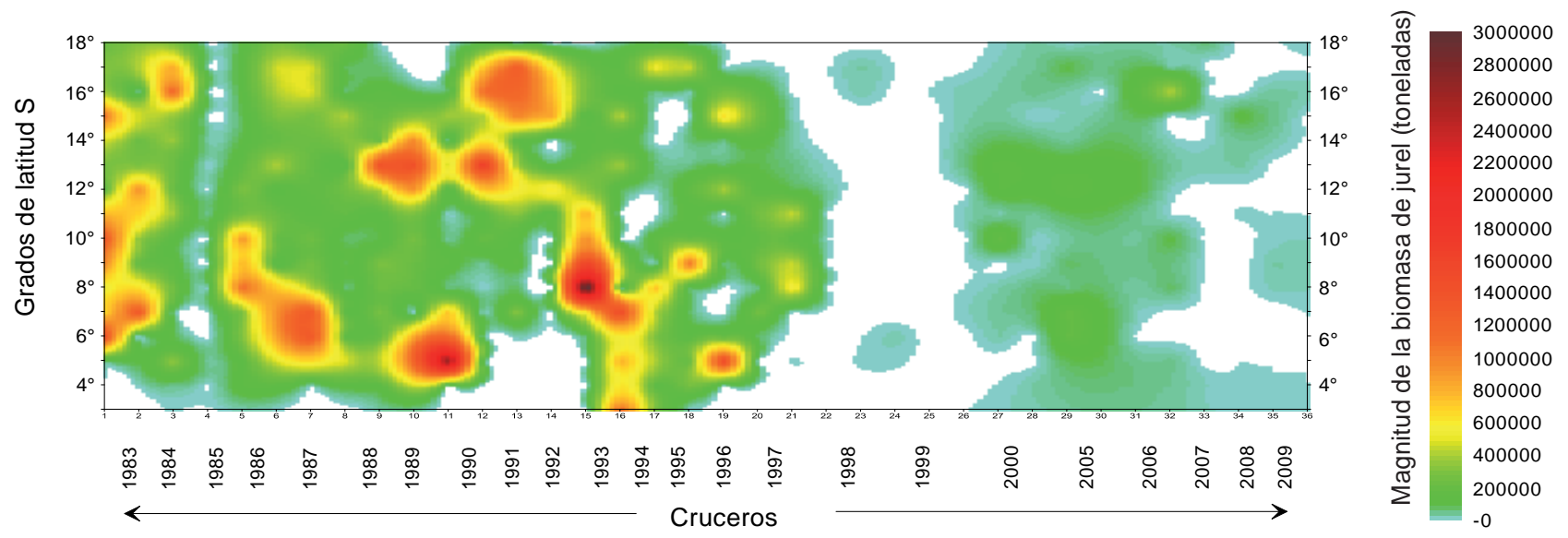

Figura 5. Distribución latitudinal de la biomasa de jurel T. murphyi estimada acústicamente entre 1983 y 2009.

Figure 5. Distribution by latitude of the estimated Jack mackerel T. murphyi acoustic biomass between 1983 and 2009. 

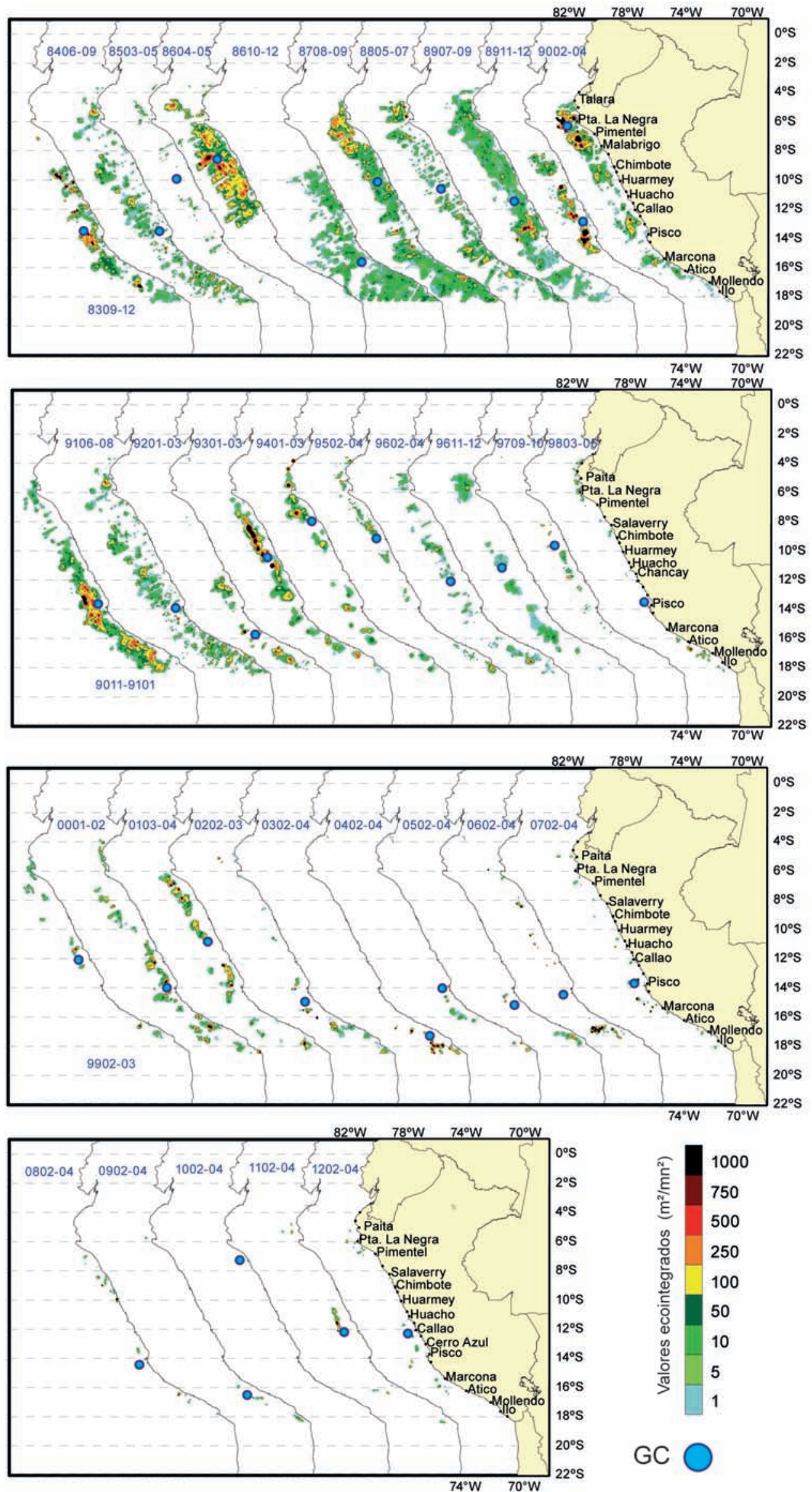

$\mathrm{GC} \bigcirc$

Figura 6. Distribución espacial y centros de gravedad (O) de las concentraciones de jurel T. murphyi entre 1983 - 2012.

Figure 6. Spatial distribution and centers of gravity (O) of Jack mackerel T. murphyi concentrations, $1983-2012$. 


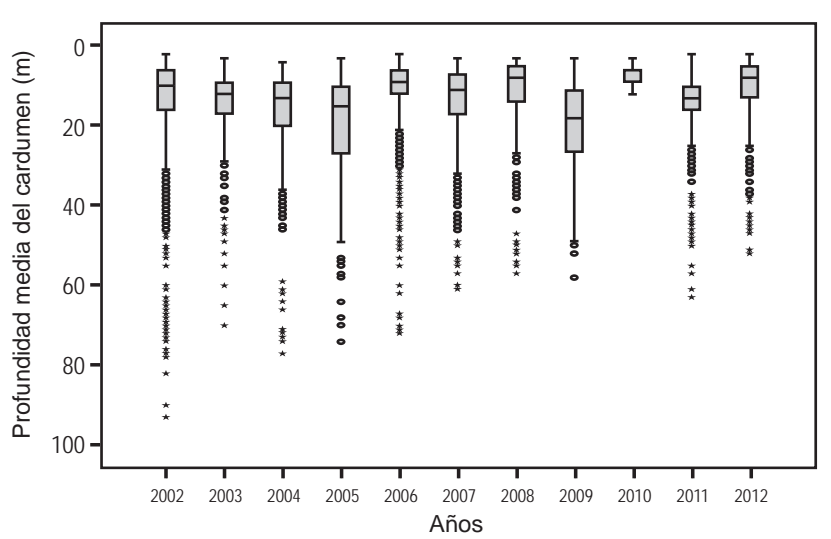

Figura 7. Profundidades medias y distribución vertical de cardúmenes de jurel T. murphyi, $2002-2012$.

Figure 7. Average depths and vertical distribution of Jack mackerel T. murphyi shoals, $2002-2012$.

entre 1983 y 2009. Se destaca que estos focos de concentración guardan buena correspondencia con la variabilidad de la configuración de las masas de agua en el mar peruano descrita por Flores et al. (2013). En esta figura se puede observar el efecto de un evento El Niño extraordinario como el de 1997-98 en la disponibilidad de jurel T. murphyi en el área evaluada, que causó la notable reducción de la biomasa acústica luego de ese evento. Es interesante anotar la concordancia de estas observaciones con los resultados de los análisis de ecología trófica presentados por Alegre et al. (2013).

Entre 1983 y 1990 se observaron concentraciones de T. murphyi en forma continua en casi todo el área evaluada (Fig. 6), con concentraciones densas al norte de Pisco (Pisco-Chimbote en 1983, Talara-Huarmey en 1986, Supe-Pisco en 1989, y Paita-Malabrigo en 1990) y dispersas en las áreas restantes. En la primavera de 1983 (Cr. 8309-12), T. murphyi se distribuyó hasta las $120 \mathrm{mn}$ de la costa, con mayores concentraciones en los $10^{\circ} \mathrm{S}$ y $14^{\circ} \mathrm{S}$. En 1988 y en 1989 alcanzó las $130 \mathrm{mn}$, y en 1990 se le detectó hasta las $140 \mathrm{mn}$.

Entre 1990 y 1998 T. murphyi ocupó pequeñas áreas de distribución en las áreas exploradas. Sólo se detectaron concentraciones densas hasta 1994, entre Pisco y Malabrigo. La distribución fue tornándose más cercana la costa, desde $120 \mathrm{mn}$ (1990) hasta $70 \mathrm{mn}$ de distancia de costa (1994). De 1995 a 1998 el jurel se observó en pequeñas concentraciones dispersas y aisladas cerca de la costa.

Entre el 2000 y 2007, T. murphyi se encontró muy disperso, con pequeñas áreas de concentración densa al sur de Pisco. Del 2008 al 2012, sus áreas de concentración continuaron siendo muy dispersas y replegadas hacia la costa, mientras que las áreas con densidades altas siguieron ausentes en el área de evaluación.

Los cambios en la distribución de T. murphyi se reflejaron también en la localización de los centros de gravedad de las concentraciones de jurel (Fig. 6), con un desplazamiento latitudinal que demuestra el dinamismo en la distribución de esta especie en el mar peruano.

En general, se observa que entre 1983 y 2012 el área ocupada por el T. murphyi fluctuó considerablemente dentro de un área total de evaluación cuya extensión no varió significativamente. A partir de 1992 se presento un cambio considerable en la extensión de las áreas de distribución de T. murphyi. Analizando
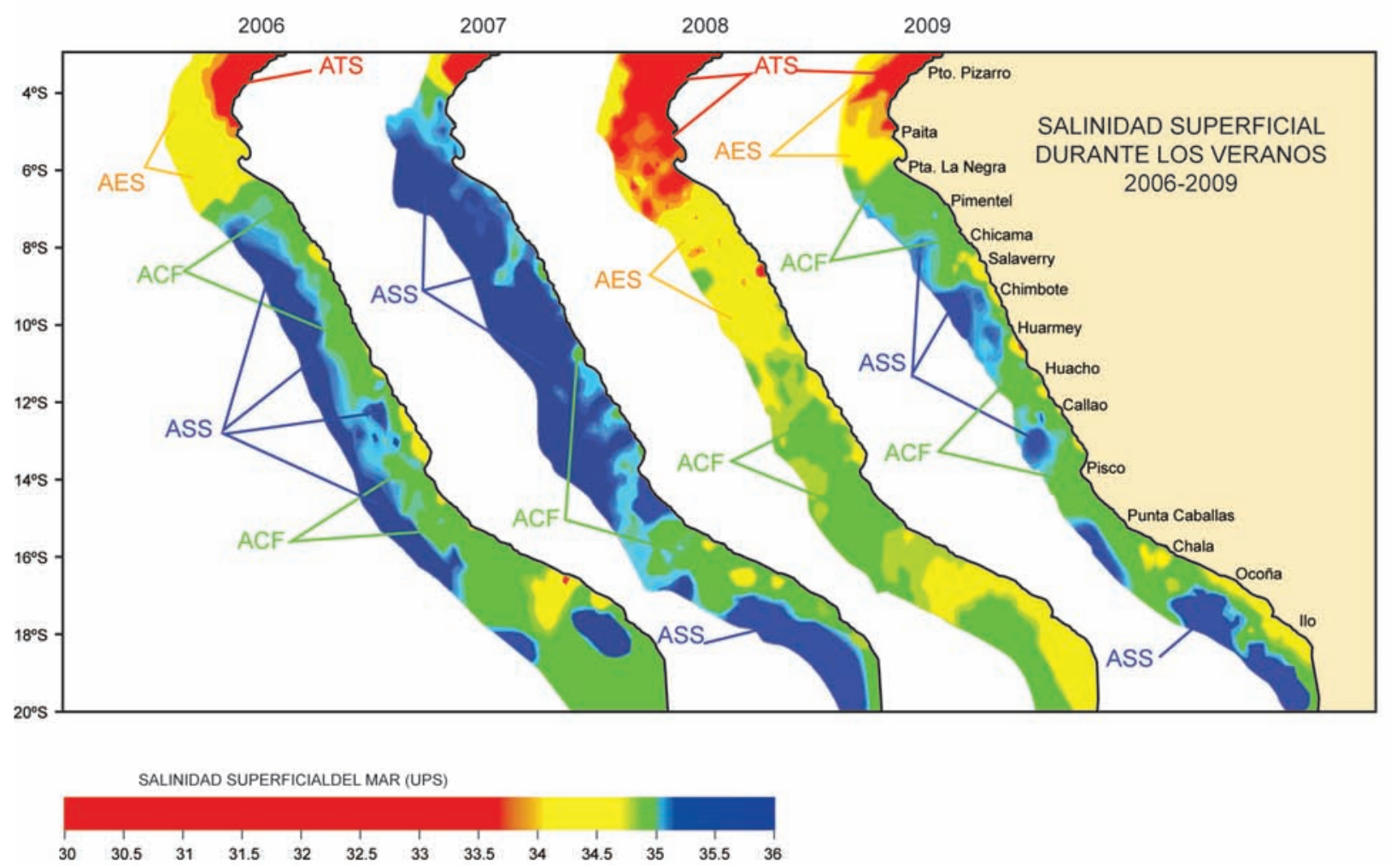

Figura 8. Distribución de la salinidad superficial y las principales masas de agua, 2006 - 2009. ATS (Aguas Tropicales Superficiales); AES (Aguas Ecuatoriales Superficiales); ACF (Aguas Costeras Frías); ASS (Aguas Subtropicales Superficiales).

Figure 8. Surface salinity and main water masses distribution, 2006-2009. ATS (superficial tropical waters or STW); AES (superficial equatorial waters or SEW); ACF (Cold coastal waters or CCW); ASS (superficial subtropical waters or SSW) 


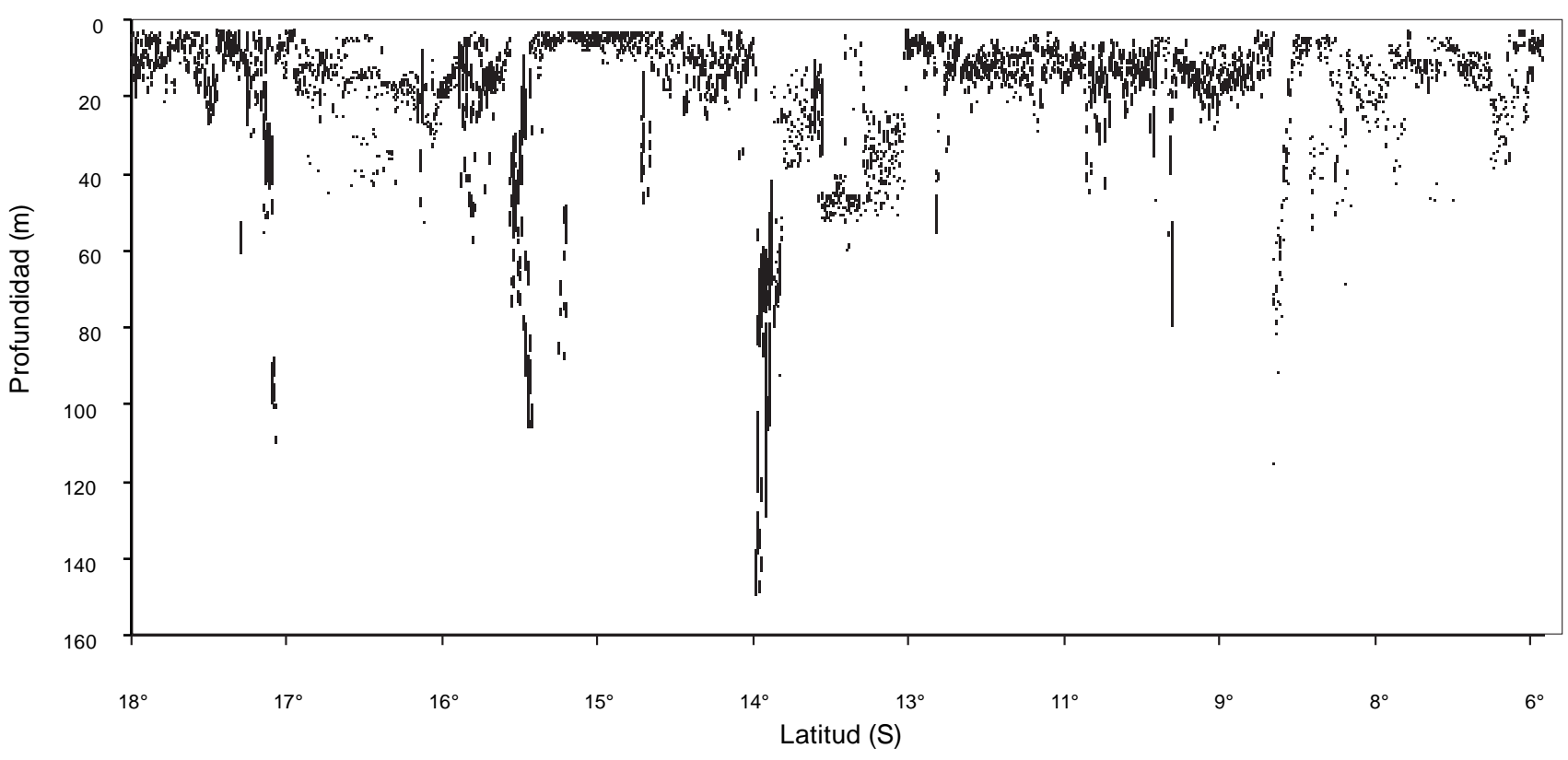

Figura 9. Profundidades medias de cardúmenes de jurel T murphyi por latitud, verano 2006.

Figura 9. Average depth of Jack mackerel T murphyi shoals by latitude, summer 2006.

la serie en dos partes y teniendo como punto de quiebre el año 1992, podemos observar que entre 1983 y 1991 el área de distribución del T. murphyi ocupó en promedio $47078 \mathrm{mn}^{2}$.

El total del área evaluada por los cruceros pelágicos fue 99045 $\mathrm{mn}^{2}$ en promedio, y el área ocupada por el T. murphyi representó en promedio el $47.5 \%$ del área total evaluada. Entre 1992 y 2012 el área total evaluada promedio disminuyó en 6.3\% (a 92788 $\mathrm{mn}^{2}$ ) sin otros cambios significativos con respecto al período anterior. Sin embargo, el promedio del área total de su distribución entre 1992 y 2012 fue de 7913 mn $^{2}$, lo que representa sólo el $8.5 \%$ del área evaluada, y el 16.8\% del área promedio ocupada por T. murphyi en el periodo anterior.
En cuanto a la distribución vertical, para el periodo 1983 a 1994, las evaluaciones hidroacústicas detectaron jurel preferentemente entre los 10 y $40 \mathrm{~m}$ de profundidad y en general el rango alcanzó hasta $240 \mathrm{~m}$ (Ganoza 1998). Icochea et al. (1989) al analizar 25 mil operaciones de pesca comercial realizadas entre febrero 1983 y marzo 1987con red de arrastre pelágica, concluyeron que cuando la Contracorriente Subsuperficial de Cromwell (CCSC) está debilitada, la pesca se concentra cerca de la superficie hasta una distancia de $200 \mathrm{mn}$ de la costa.

Podemos interpretar que cuando la CCSC se fortalece, la pesca se concentra dentro de las $60 \mathrm{mn}$ con migraciones verticales hasta $300 \mathrm{~m}$ en el día y un acercamiento a la superficie en la noche. En condiciones normales, T. murphyi fue detectado

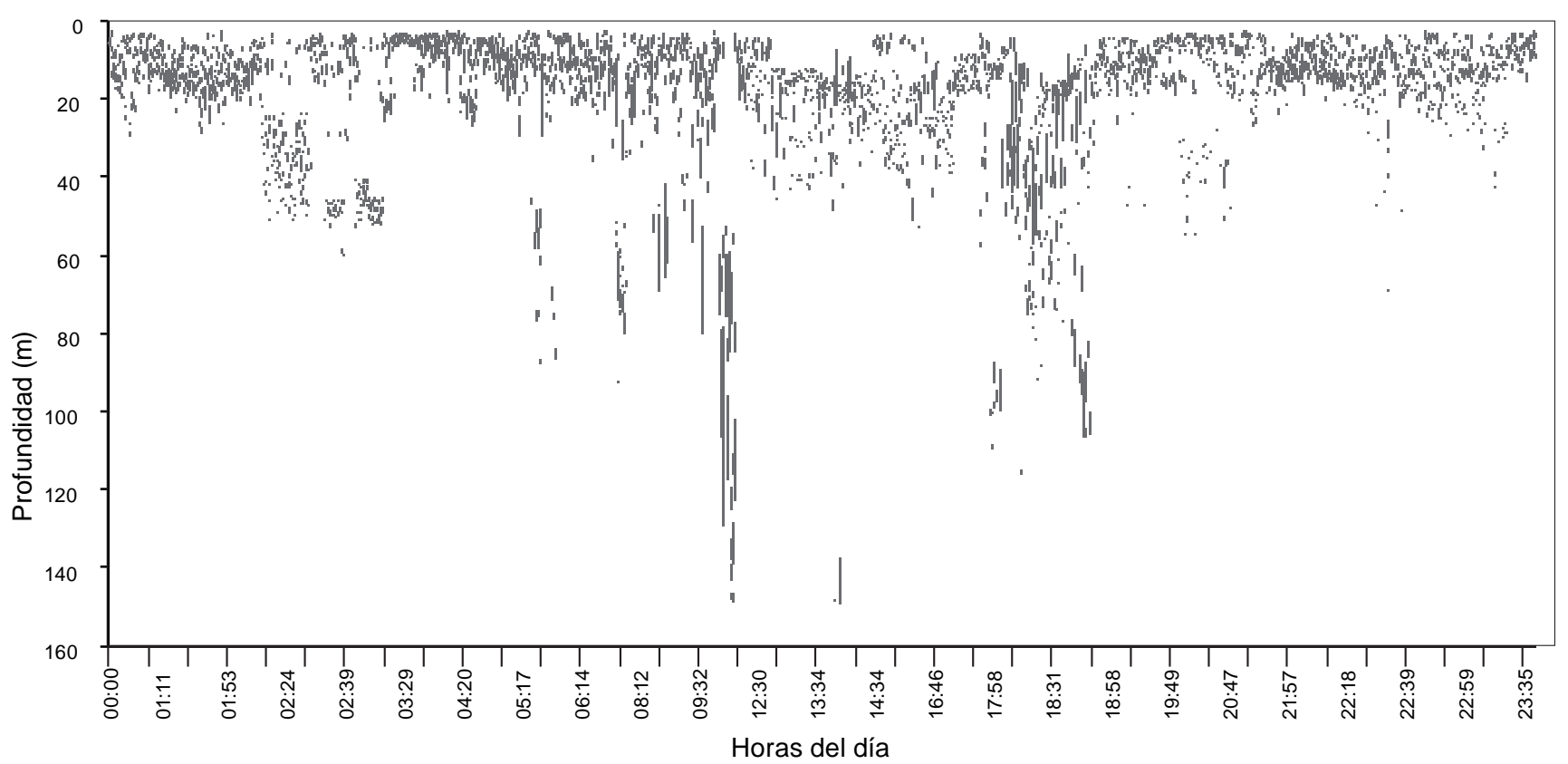

Figura 10. Distribución vertical de cardúmenes de jurel T. murphyi según horas del día, verano 2006.

Figure 10. Jack mackerel T. murphyi vertical shoals distribution by hours during summer 2006 
con mayor frecuencia por encima de los $50 \mathrm{~m}$ de profundidad. En eventos El Niño, T. murphyi se profundiza hasta $200 \mathrm{~m}$ con desplazamientos verticales desde la superficie hasta cerca a los $350 \mathrm{~m}$ de profundidad (Dioses \& Niquen, 1988).

En general, durante el periodo 2002 al 2012, la profundidad media de los cardúmenes de jurel se ubicó alrededor de los $15 \mathrm{~m}$, sin embargo durante este periodo observamos que T. murphyi disminuyó su rango de distribución vertical (Fig. 7). Entre 2002 y 2006 los cardúmenes se encontraron principalmente a profundidades que alcanzaron $l o s 70 \mathrm{~m}$ y en algunos casos llegaron hasta 150 m. Pero entre 2007 y 2012 los cardúmenes se encontraron preferentemente en los primeros $40 \mathrm{~m}$ profundidad.

En el mar peruano, la distribución de T. murphyi muestra una estrecha relación con variables ambientales como la temperatura y la salinidad (Fig. 8). Varios estudios y análisis han sustentado el rol de la Zona Mínima de Oxigeno (ZMO) como regulador de los procesos claves de la productividad y la distribución de los recursos pelágicos (Bertrand et al 2010). Esta relación es notoria, particularmente durante eventos El Niño o La Niña, cuando se observa gran fluctuación de estas variables y T. murphyi muestra gran capacidad de respuesta modificando tanto su distribución horizontal como vertical en la época de estos eventos (Figs. 6, $7,9$ y 10$)$.

Se ha observado que la distribución vertical de las concentraciones de T. murphyi cambia según la latitud y horas del día. En la Figura 9 se puede apreciar que en el verano de 2006 los cardúmenes alcanzan mayor profundidad en la zona centro-sur, especialmente en el paralelo $14^{\circ} \mathrm{S}$. Respecto a las horas, existe una tendencia a que los cardúmenes vayan más hacia la superficie durante la noche y las primeras horas del día debido a su ciclo alimenticio y la agregación de macro-zooplancton en forma de cordón en las noches (Fig. 10).

Conclusiones.- La biomasa de T. murphyi estimada por el método de evaluación hidroacústica tuvo dos valores máximos: el primero en 1983, con 8512674 t; y el otro en 1993, con 8471096 t. Luego se observa una tendencia decreciente, con un estimado de biomasa de solamente 1239 t para el año 2010.

Los estimados de biomasa del T. murphyi dentro del área evaluada disminuyeron drásticamente a partir de 1992, año en el cual se da un punto de quiebre en su distribución y concentración en aguas peruanas. La misma tendencia se observo en la extensión de sus áreas de concentración.

Trachurus murphyi muestra una marcada variabilidad en su abundancia y sus áreas de distribución entre 1983 - 2012. Hasta antes de 1994, las concentraciones densas se encontraron al norte de Pisco, mientras que de 1995 al 2012 las concentraciones se reportaron al sur en concentraciones aisladas y muy reducidas.

Frente a las costas de Perú, la distribución del T. murphyi muestra una estrecha interrelación con parámetros ambientales como la temperatura, la salinidad y el oxígeno.

Tanto en su distribución horizontal como vertical, la especie muestra una gran capacidad de adaptación a las condiciones de los eventos ENSO que afectan los tipos, formas, dinámica de cardumen y dinámica nictemeral.
Las evaluaciones de T. murphyi han estado condicionadas a la distribución de la anchoveta E. ringens que tiene una distribución más costera y es muy probable que subestimen las verdaderas existencias de T. murphyi frente a las costas peruanas en la medida que parte de la población se distribuye a mayor distancia de la costa en aguas peruanas o también en alta mar.

\section{Literatura citada}

Alegre A., P. Espinoza \& M. Espino. 2013. Ecología trófica del jurel Trachurus murphyi en el Perú entre 1977-2011. En: Csirke J., R. GuevaraCarrasco \& M. Espino (Eds.). Ecología, pesquería y conservación del jurel (Trachurus murphyi) en el Perú. Rev. peru. biol. número especial 20(1): $075-082$

Ayón P. \& J. Correa. 2013. E Variabilidad espacial y temporal de larvas de jurel Trachurus murphyi en el Perú entre 1966-2010. En: Csirke J., R. Guevara-Carrasco \& M. Espino (Eds.). Ecología, pesquería y conservación del jurel (Trachurus murphyi) en el Perú. Rev. peru. biol. número especial 20(1): $083-086$

Barbieri M.A., V. Correa \& J. Córdova. 2005. Pesca de investigación: “Determinación de la distribución espacial de jurel entre la $\mathrm{V}$ y X Regiones”. Otońo 2005. Informe final - Subpesca. Instituto de Fomento Pesquero. Chile.

Barbieri M.A, V. Correa \& J. Córdova. 2004. Pesca de investigación “Determinación de la distribución espacial de jurel entre la $\mathrm{V}$ y X Regiones. Invierno 2004. Informe final -Subpesca. Instituto de Fomento Pesquero. Chile. 20 pp. (más tablas y figuras).

Bazigos G.P. 1975. The statistical efficiency of echo surveys with special reference to Lake Tanganyika. FAO Fisheries Technical Paper 139. 43 pp.

Bertrand, A., Ballón, M \& A. Chaigneau. 2010 Acoustic Observation of Living Organisms Reveals the Upper Limit of the Oxygen Minimum Zone. PLoS ONE S(4): e10330. doi:10.1371/journal.pone.0010330.

Csirke J. 1995. Fluctuations in abundance of small and mid-sized pelagic. Science Marine 59(3-4): 481-490.

Castillo, P.R., S. Peraltilla, A. Aliaga, M. Flores, M. Ballón, J. Calderón \& M. Gutiérrez, 2009. Protocolo técnico para la evaluación acústica de las áreas de distribución y abundancia de recursos pelágicos en el mar peruano. Versión 2009. Informe Inst. Mar Perú, 36(1-2): 7-28.

Dioses T. \& M. Niquen, 1988. Aspectos generales sobre la biología y pesquería de recurso jurel (Trachurus murphyi) en Perú. En FORUM: Características en el diseño de una embarcación ideal para la captura de especies pelágicas 7. PESCA PERU. 36 pp.

Flores R., M. Espino, G. Luque \& J. Quispe. 2013. Patrones de variabilidad ambiental en el mar peruano. En: Csirke J., R. Guevara \& M. Espino (Eds.). Ecología, pesquería y conservación del jurel (Trachurus murphyi) en el Perú. Rev. peru. biol. número especial 20(1): 021 - 028

Foote K. 1987. Fish target strengths for use in echo integrator surveys. Journal of the Acoustical Society of America 82:981-987. DOI: $10.1121 / 1.395298$

Ganoza F. 1998. Abundancia y comportamiento del jurel (Trachurus picturatus murphyi) a través de prospecciones acústicas de 1983 a 1994. Inf. Inst. Mar Perú. 136: 23-47.

Gutiérrez M. \& S. Peraltilla. 1999. Aplicación de un sistema de información geográfica y de la carta electrónica isoparalitoral en las evaluaciones hidroacústicas de las biomasas de recursos pesqueros en el litoral peruano. Inf. Inst. Mar Perú 146: 25-29.

Gutiérrez M. 1997. Aplicación de software de interpolación en las evaluaciones hidroacústicas de la biomasa y distribución de los recursos pelágicos. Inf. Prog. Inst. Mar Perú 67: 21-30.

Gutiérrez M., N. Herrera \& D. Marín. 1999. Distribución y abundancia de anchoveta y otras especies pelágicas entre los eventos El Niño de 1982-83 y 1997-98. Bol. IMARPE, 18(1-2): 77-88.

Icochea L., A. Chipollini \& M. Niquen. 1989. Análisis de pesquería de arrastre pelágica en la costa peruana durante 1983-1987 y su relación con el medio ambiente. Memorias del Simposio Internacional de los Recursos Vivos y las Pesquerías en el Pacífico Sudeste. Viña del mar, 9-13 mayo, 1988.

Johannesson K. \& A. Robles. 1977. Echo surveys of Peruvian anchoveta. Rapp. P.-V. Réu. Cons. Int. Explor. Mer. 170: 237-244.

Johannesson K. \& R. Vilchez. 1981. Application and some results of echointegration methods of monitoring Peruvian anchovy resources. In: J. Suomala (Ed.). Meeting on hydroacoustical methods for the estimation of marine fish populations, 25-19 June, 1979. Charles Stark Draper Laboratory Inc. Cambridge, MA. Vol. 2b: pp. 756-816. 
Jordán J. 1983. Variabilidad de los recursos pelágicos en el Pacífico Sudeste. En G.D. Sharp y J. Csirke (Eds.). Actas de la Consulta de Expertos para examinar los cambios en la abundancia y composición por especies de recursos de peces neríticos. San José, Costa Rica, 18-29 abril 1983. Una reunión preparatoria para la Conferencia Mundial de la FAO sobre Ordenación y Desarrollo Pesqueros. FAO Fish.Rep./ FAO Inf. Pesca (291), Vol. 2: pp. 113-129.

MacLennan D. \& J. Simmonds. 1992. Fisheries Acoustics. London, Chapman and Hall Eds. 325pp.

Serra J.R. 1991. Important life history aspects of the chilean Jack mackerel,Trachurus symmetricus murphyi. Invest. Pesq. (Chile) 36:67-83.

Serra J. R. 1983. Changes in the abundance of pelagic resources along the Chilean coast. In Proceedings of the Expert Consultation to Examine Changes in Abundance and Species Composition of Neritic Fish Resources. San Jose, Costa Rica, April 1983. Sharp, G.D. and J. Csirke (Eds). FAO Fish. Rep. 291 (2): 255-284.
Simmonds E.J. \& D.N. MacLennan. 2005. Fisheries acoustics, theory and practice. Blackwell Science, 472 pp.

SIMRAD. 1992. Instruction Manual, Simrad EK 500 scientific echo sounder. P217E. Calibration of the EK 500 P2260E. Simrad Norge AS. Norway: $15-16$

Zuta S., I. Tsukayama \& R. Villanueva. 1983. El ambiente marino y las fluctuaciones de las principales poblaciones pelágicas de la costa peruana. En G.D. Sharp \& J. Csirke (Eds.). Actas de la Consulta de Expertos para examinar los cambios en la abundancia y composición por especies de recursos de peces neríticos. San José, Costa Rica, 18-29 abril 1983. Una reunión preparatoria para la Conferencia Mundial de la FAO sobre ordenación y desarrollo pesqueros. FAO Fish. Rep./ FAO Inf. Pesca (291), Vol. 2: pp. 179-253. 